\title{
Nanostructured Materials: Formation, Characterization, and Properties-Latest Advances in 1D, 2D, and 3D Nanostructures
}

\author{
Luís Cunha, ${ }^{1}$ Denis Chaumont, ${ }^{2}$ and Aldo Craievich ${ }^{3}$ \\ ${ }^{1}$ Centro de Física, Universidade do Minho, Campus de Gualtar, 4710-057 Braga, Portugal \\ ${ }^{2}$ Laboratoire ICB, Université de Bourgogne, 9 avenue Alain Savary, BP 47 870, 21078 Dijon Cedex, France \\ ${ }^{3}$ Instituto de Física, Universidade de São Paulo, Rua do Matão Travessa R 187, 05508-900 São Paulo, SP, Brazil \\ Correspondence should be addressed to Luís Cunha; lcunha@fisica.uminho.pt
}

Received 7 April 2014; Accepted 7 April 2014; Published 17 April 2014

Copyright (C) 2014 Luís Cunha et al. This is an open access article distributed under the Creative Commons Attribution License, which permits unrestricted use, distribution, and reproduction in any medium, provided the original work is properly cited.

\begin{abstract}
“There's plenty of room at the bottom," said Richard P. Feynman, during a talk in December 29, 1959. These words are considered the beginning of the nanoera, despite a paper published by Radushkevich and Lukyanovich, in 1952, showing graphitic carbon fibres with 50 nanometers in diameter. In any case the door of the world of nanoscience and nanotechnology was opened. Feynman's premonitory words became a reality and the nanoworld never stopped evolving. The research done in this field allows developing novel nanostructures, which sooner or later will have an impact on society and will promote the development of entrepreneurial activities for the use of advanced technologies.

The main focus of this special issue aims at contributing to the dissemination of new developments and original ideas related to the production, characterization, and applications of nanostructures to several fields such as nanomedicine, nanoelectronics, manipulation at nanoscale, optics, and photonics. The accepted articles cover different topics, namely, preparation of nanoparticles and nanostructures, surface plasmon resonance (SPR) effect, surface enhanced Raman scattering (SERS), preparation of SERS active surfaces, effect of annealing temperature on the magnetic properties of $\mathrm{Nd}$ $\mathrm{Fe}-\mathrm{B}$ nanocomposites, and biotransformation.

The article "Nanocrystalline Al composites from powder milled under ammonia gas flow" by J. Cintas et al. describes the synthesis of hard and thermally stable $\mathrm{Al}$ composites, prepared by powder milling $\mathrm{Al}$ in the presence of ammonia flow, followed by the sintering of milled powders. The Al grain growth is constrained to a maximum size of $45 \mathrm{~nm}$.

"Size controlled synthesis of FeCo alloy nanoparticles and study of the particle size and distribution effects on magnetic
\end{abstract}

properties" is the title of the article by A. Shokuhfar and S. S. S. Afghahi, in which the authors describe the quaternary microemulsion system used to control the production of FeCo alloy nanoparticles and the effect of the processing parameters on their size and size distribution. The influence of the size of the particles on the magnetic properties was also investigated.

Magnetic properties were also the subject of the paper "Influence of annealing temperature on the magnetic properties of rapidly quenched $(\mathrm{Nd}, \mathrm{Pr})_{2}-(\mathrm{Fe}, \mathrm{Co}, \mathrm{Ga}, \mathrm{Ti}, \mathrm{C})_{14} \mathrm{~B} / \mathrm{\alpha}-\mathrm{Fe}$ nanocomposite ribbons," by R. Sabbaghizadeh et al. This study describes the production of nanocomposite ribbons, with grain size lower than $50 \mathrm{~nm}$, by melt spinning and the optimized postannealing process that leads to the best magnetic properties.

Two articles dealt with the production of substrates for surface enhanced Raman scattering (SERS) analysis: "Fabrication of SERS active surface on polyimide sample by excimer laser irradiation" by T. Csizmadia et al. and "Synthesis of dendritic silver nanoparticles and their applications as SERS substrates" by J. Yu and X. Zhou.

T. Csizmadia et al. used an excimer laser to irradiate a polyimide foil, before coating it with Ag, by pulsed laser deposition, using different fluences. The study of the activity of the surface for SERS was done by using a rhodamine $6 \mathrm{G}$ aqueous solution.

A rhodamine $6 \mathrm{G}$ aqueous solution was also used by J. Yu and $\mathrm{X}$. Zhou to study the possibility of using silver nanoparticles, with dendritic morphology, deposited onto a Ag crystal, for SERS. The silver nanoparticles, with sizes ranging from 20 to $30 \mathrm{~nm}$, were prepared by electrodeposition. 
Both articles conclude that the substrates exhibit meaningful results concerning their potential to be used for SERS.

A. Espíndola-Gonzalez et al. authored the article entitled "Structural characterization of silica particles extracted from grass Stenotaphrum secundatum: biotransformation via annelids." This paper describes the characterization of silica particles extracted by biotransformation from St. Augustine grass. These particles were compared with silica particles extracted directly from the grass and significant differences in size and structure were detected.

In short, this issue contains original contributions to synthesis, characterization, and properties of nanoparticles and nanostructures. We expect that the readers working in the field of nanoscience and nanotechnology will find in the different articles new motivating ideas useful for their ongoing investigations.

Luís Cunha

Denis Chaumont Aldo Craievich 

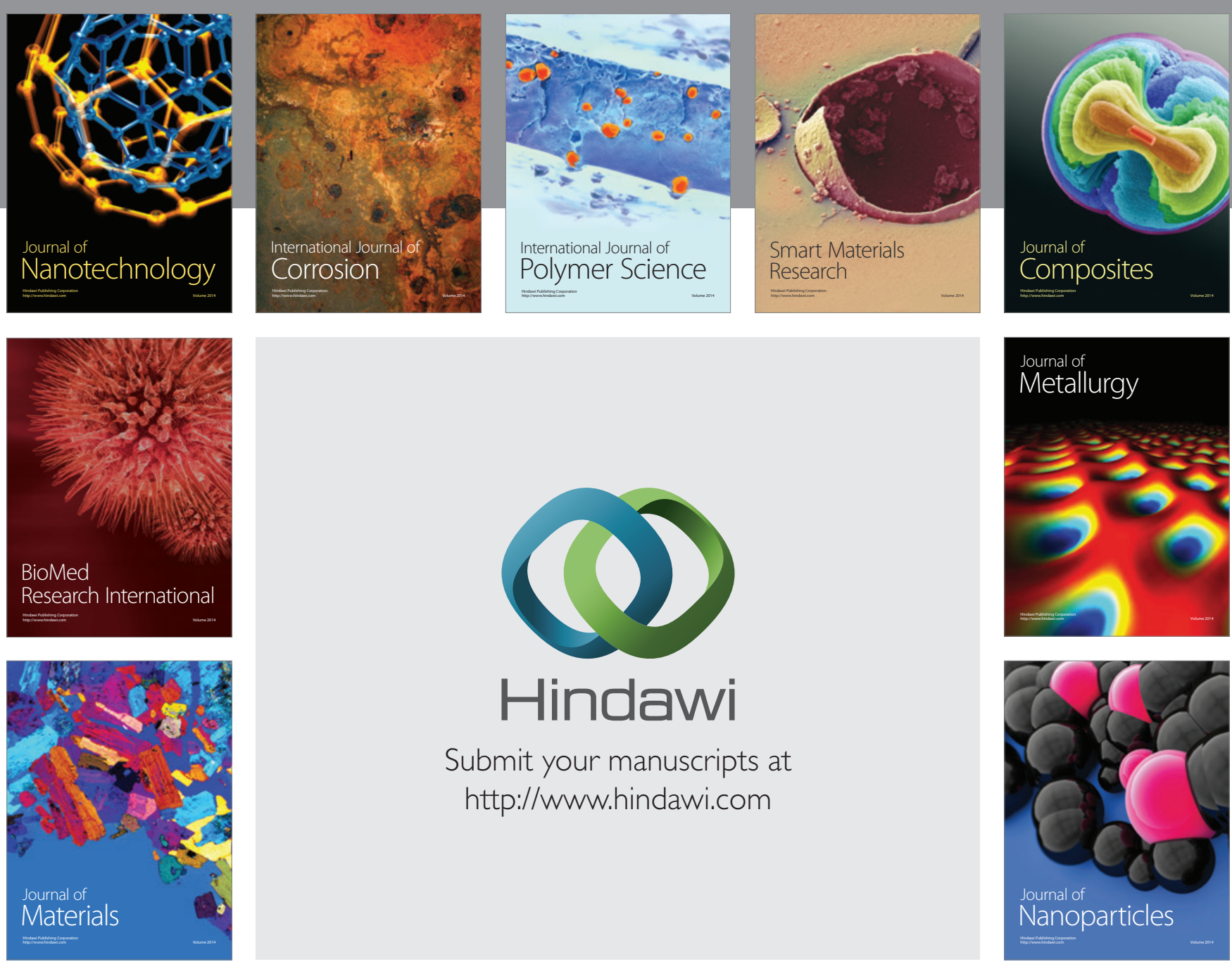

Submit your manuscripts at http://www.hindawi.com
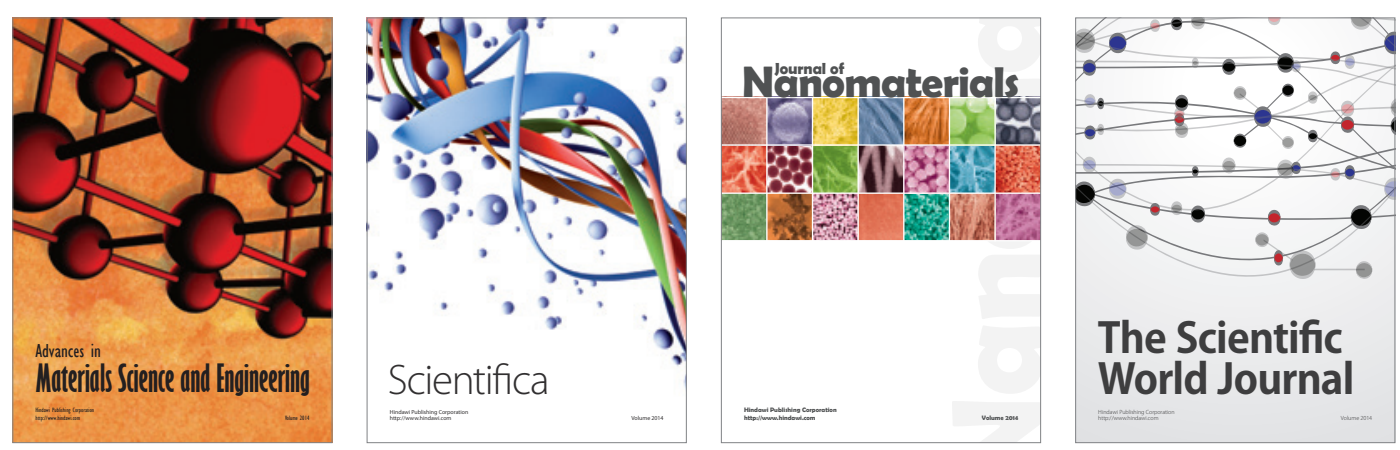

\section{The Scientific World Journal}
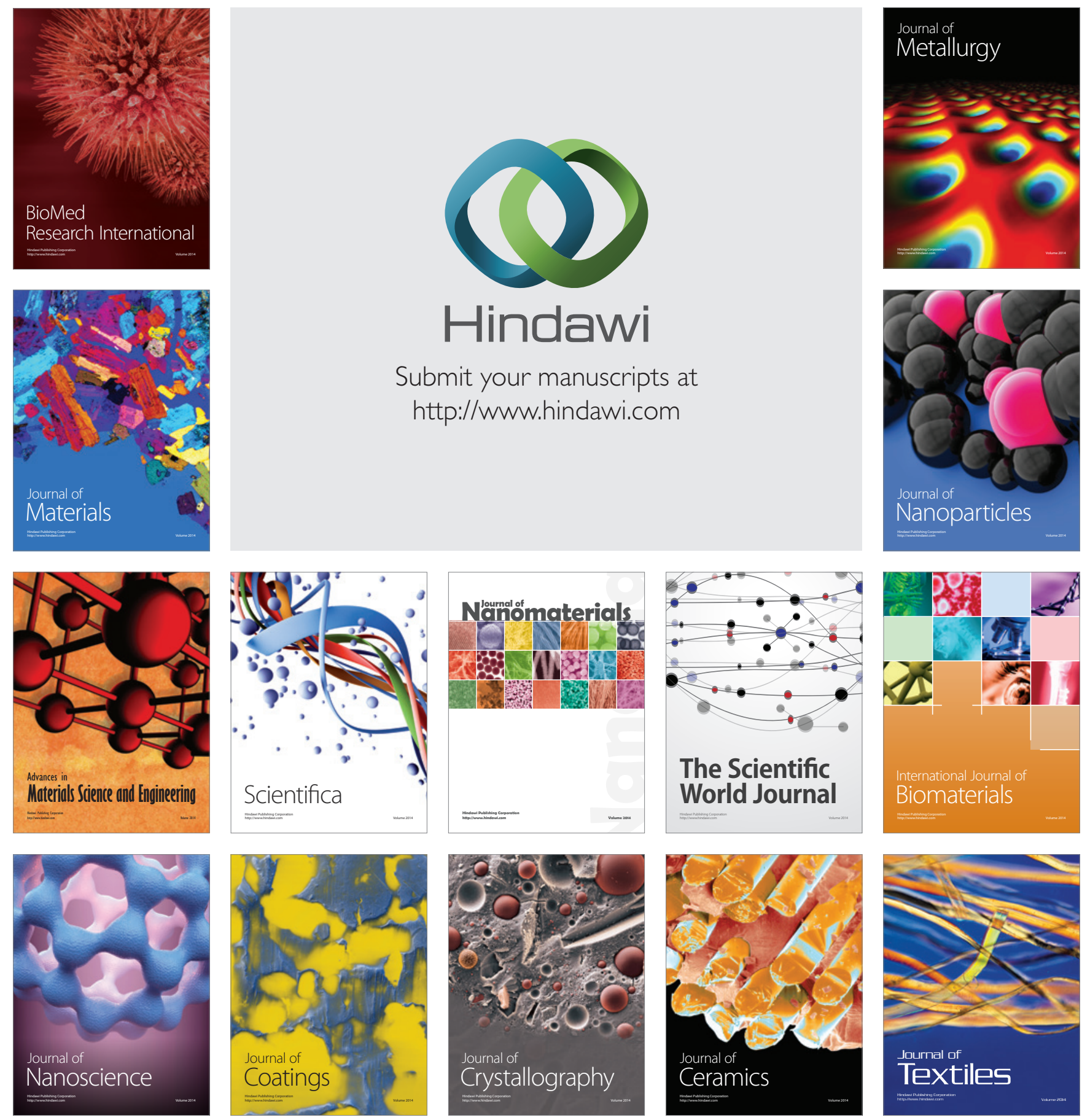